\title{
Semiclassical evaluation of average nuclear one- and two-body matrix elements
}

\author{
X. Viñas, ${ }^{1}$ P. Schuck,${ }^{2}$ M. Farine,${ }^{3}$ and M. Centelles ${ }^{1}$ \\ ${ }^{1}$ Departament d'Estructura i Constituents de la Materia, Facultat de Física, Universitat de Barcelona, Diagonal 647, 08028 Barcelona, \\ Spain \\ ${ }^{2}$ Institut de Physique Nucléaire, IN2P3-CNRS, Université Paris-Sud, 91406 Orsay-Cédex, France \\ ${ }^{3}$ Consulat Général de France à Canton, 339 Huan Shi Dong Lu, 510098 Guangzhou, Canton, China
}

(Received 21 June 2002; revised manuscript received 26 February 2003; published 15 May 2003)

\begin{abstract}
Thomas-Fermi theory is developed to evaluate nuclear matrix elements averaged on the energy shell, on the basis of independent particle Hamiltonians. One- and two-body matrix elements are compared with the quantal results, and it is demonstrated that the semiclassical matrix elements, as function of energy, well pass through the average of the scattered quantum values. For the one-body matrix elements it is shown how the ThomasFermi approach can be projected on good parity and also on good angular momentum. For the two-body case, the pairing matrix elements are considered explicitly.
\end{abstract}

DOI: 10.1103/PhysRevC.67.054307

PACS number(s): 21.10.Dr, 21.60.-n, 31.15.Gy

\section{INTRODUCTION}

The solution of the nuclear many-body problem presents a formidable challenge. Not only bare and effective nucleonnucleon forces are not completely known, but still for those given as granted, one has to solve the many-body problem of a highly quantal, strongly interacting, self-bound, and therefore inhomogeneous Fermi system. Over the years, semiclassical techniques have helped to solve this problem, for instance, in regard to the latter aspect. In practice, it is mainly the Thomas-Fermi (TF) method and its extensions for the description of nuclear ground-state properties which has been considered (see Ref. [1] and references therein). The nuclear density and kinetic energy density are the main ingredients of this approach.

The semiclassical approximation often gives a direct physical insight, yielding the shell average of the quantities under consideration and providing their main trend (which, in certain cases, may be obscured by strong shell fluctuations). A known example is the nuclear binding energy which coincides with the liquid drop part in the semiclassical approach. Another quantity of longstanding interest is the average single-particle level density. It is well known that the TF approximation to the level density (including $\hbar$ corrections) coincides analytically with the Strutinsky averaged quantal level density for the harmonic oscillator (HO) potential [2]. First performing the quantal calculation and then the average is more cumbersome than calculating the shell average directly via the TF method. The technical advantage of the latter becomes significant in the deformed case [2] or, for instance, when one wants to go beyond the independent particle description to include correlations [3]. The TF approach is also very helpful for the calculation of surface and curvature energies. Actually, the latter quantity can only be correctly extracted in a semiclassical procedure [4].

In general, however, we believe that the true virtue of the TF method shows up not only in calculating average properties in the independent particle approximation, where it can replace the results obtained through the more cumbersome Strutinsky method [5], but rather in many-body applications going beyond the mean field or independent particle picture where a straightforward quantum solution for finite systems may reach its limits. A case where we treated correlation effects in TF approximation was, as already mentioned, the level density parameter [3]. Pairing correlations in finite nuclei have also already successfully been treated in the past [6]. This is one of the aspects which we shall consider again in this work in more detail.

In this work we want to discuss on an aspect of the Thomas-Fermi theory which in the past has been exploited only very little. This concerns the evaluation of matrix elements averaged over a certain energy interval that may be typically of the order of $\hbar \omega$, i.e., the separation of major shells. It must be pointed out that this is, to our knowledge, the first attempt to evaluate not only one-body but also twobody matrix elements in the TF approximation. This semiclassical calculation provides the smoothly varying part of the matrix elements dropping the shell effects according to the idea of the Strutinsky averaging method [7]. We will describe several tests of the accuracy of the TF method for on-shell densities. In the first part we will develop our approach for the matrix elements of single-particle operators, for given parity and angular momentum. This goes along similar lines already developed in the domain of systems with chaotic behavior [8-11]. In the second part we will address our main objective, which is to show that the method also works for two-body matrix elements. Some preliminary results have been published previously in Ref. [12]. As a specific example, we will treat the pairing matrix elements.

Let us give a short summary of the approach we are going to develop. Consider, for example, the expectation value of a single-particle operator $\hat{O}$ in some shell model state $|\nu\rangle$ :

$$
O_{\nu}=\langle\nu|\hat{O}| \nu\rangle=\operatorname{Tr}[\hat{O}|\nu\rangle\langle\nu|]
$$

Instead of knowing the $O_{\nu}$ quantum state by a quantum state it may sometimes be advantageous and instructive to only know how matrix element (1) changes as a function of energy. We therefore introduce a single-particle matrix element averaged over the energy shell:

$$
O(E)=\operatorname{Tr}\left[\hat{O} \hat{\rho}_{E}\right]
$$


where we call $\hat{\rho}_{E}$ the density matrix on the energy shell. It is related with the so-called spectral density matrix and will be defined immediately below.

The spectral density matrix $\delta(E-\hat{H})$ has the characteristic discontinuous behavior due to the quantization of the eigenvalues of the single-particle Hamiltonian $\hat{H}$. It can be written, however, as a sum of a smooth part $\widetilde{\delta}(E-\hat{H})$ and of a strongly oscillating part, i.e., $\delta(E-\hat{H})=\widetilde{\delta}(E-\hat{H})$ $+\delta_{\text {osc }}(E-\hat{H})[1,13,14]$. Analogously, the single-particle level density $g(E)=\operatorname{Tr}[\delta(E-\hat{H})]=\Sigma_{\nu} \delta\left(E-\varepsilon_{\nu}\right)$ is obtained as a sum of two terms: $g(E)=\tilde{g}(E)+g_{\text {osc }}(E)$, where $\tilde{g}$ and $g_{\text {osc }}$ stand for the smooth and the rapidly fluctuating contributions, respectively. Using the smooth $\widetilde{\delta}(E-\hat{H})$ and $\widetilde{g}(E)$, we define the density matrix averaged on the energy shell as

$$
\hat{\rho}_{E}=\frac{1}{\widetilde{g}(E)} \widetilde{\delta}(E-\hat{H})=\frac{1}{\widetilde{g}(E)} \sum_{\nu} \widetilde{\delta}\left(E-\varepsilon_{\nu}\right)|\nu\rangle\langle\nu| .
$$

It is therefore a smooth function of $E$. The smeared level density $\widetilde{g}(E)$ (per spin and isospin in this paper) in the denominator of expression (3) ensures the right normalization of $\hat{\rho}_{E}$, since $\tilde{g}(E)=\operatorname{Tr}[\widetilde{\delta}(E-\hat{H})]$.

The smooth quantities entering Eq. (3) are to be evaluated in some continuum limit $[13,14]$. This is the case, for example, when one introduces the Strutinsky averaging procedure $[5,7]$ or alternatively (and this is the approach we adopt in this work), it can be done by replacing $\hat{H}$, the independent-particle (mean field) Hamiltonian, by its classical counterpart $H_{c l}$ that corresponds to the TF approximation [1]. Such an approximation has been used very early by Migdal [15] and later, as already mentioned, in the context of chaotic motion dynamics [8-11]. Recently, we have employed it to describe Bose condensates in traps [16], but we are not aware of any systematic use in the context of nuclear physics. The approach is not limited to the evaluation of expectation values of single-particle operators. Also the average behavior of two-body matrix elements can be calculated. For instance, the semiclassical evaluation of the average pairing matrix element,

$$
\begin{aligned}
v\left(E, E^{\prime}\right)= & \frac{1}{\widetilde{g}(E) \widetilde{g}\left(E^{\prime}\right)} \sum_{\nu, \nu^{\prime}} \widetilde{\delta}\left(E-\varepsilon_{\nu}\right) \widetilde{\delta}\left(E^{\prime}-\varepsilon_{\nu^{\prime}}\right) \\
& \times\left\langle\Phi(\nu, \bar{\nu})|v| \Phi\left(\nu^{\prime}, \bar{\nu}^{\prime}\right)\right\rangle,
\end{aligned}
$$

where $|\Phi(\nu, \bar{\nu})\rangle$ is an antisymmetric normalized two-body state constructed out of a state $|\nu\rangle$ and its time-reversed state $|\bar{\nu}\rangle$, can be of great practical interest and shall be considered in this work. As it is known [1,2], the Strutinsky method averages the density matrix over an energy interval corresponding roughly to the distance between two major shells. Implicitly the same holds if the equivalent Wigner-Kirkwood expansion (TF approximation at lowest order) is used for $\hat{\rho}_{E}$.

Our paper is organized as follows. In the following section, the Wigner function on the energy shell is introduced and applied to the evaluation of some semiclassical one-body and two-body matrix elements of physical interest. Our conclusions are given in the last section.

\section{WIGNER FUNCTION ON THE ENERGY SHELL}

As stated in Eq. (3) of the Introduction, we are interested in the density matrix $\hat{\rho}_{E}$ on the energy shell. Consider the Wigner transform [1] of density matrix (3), namely,

$$
f_{E}(\boldsymbol{R}, \boldsymbol{p})=\int d \boldsymbol{s} e^{-i \boldsymbol{p} s / \hbar}\left\langle\boldsymbol{R}+\boldsymbol{s} / 2\left|\hat{\rho}_{E}\right| \boldsymbol{R}-\boldsymbol{s} / 2\right\rangle,
$$

where

$$
\boldsymbol{R}=\left(\boldsymbol{r}+\boldsymbol{r}^{\prime}\right) / 2, \quad \boldsymbol{s}=\boldsymbol{r}-\boldsymbol{r}^{\prime}
$$

are the centroid and relative coordinates, respectively. In order to obtain the TF approximation plus $\hbar$ corrections to the Wigner function on the energy shell (5), it is convenient to differentiate with respect to $E$ the Wigner-Kirkwood expansion of the full single-particle one-body density matrix $\hat{\rho}$ $=\Theta(E-\hat{H})$, which is amply given in the literature [1]. Up to order $\hbar^{2}$, the result is

$$
\begin{aligned}
f_{E}(\boldsymbol{R}, \boldsymbol{p})= & \frac{1}{\tilde{g}(E)}\left[\delta\left(E-H_{c l}\right)-\frac{\hbar^{2}}{8 M} \nabla^{2} V \delta^{\prime \prime}\left(E-H_{c l}\right)\right. \\
& +\frac{\hbar^{2}}{24 M}\left[(\boldsymbol{\nabla} V)^{2}+\frac{1}{M}(\boldsymbol{p} \cdot \boldsymbol{\nabla})^{2} V\right] \delta^{\prime \prime \prime}\left(E-H_{c l}\right) \\
& \left.+O\left(\hbar^{4}\right)\right]
\end{aligned}
$$

One should realize that $\widetilde{g}(E)$ also contains $\hbar$ corrections and that, strictly speaking, in order to get a consistent expansion of $f_{E}$ one should also take into account the $\hbar$ expansion of $\tilde{g}(E)$ and then correctly sort out relation (6) to order $\hbar^{2}$ (see also comments at the end of Sec. II A).

The first term in Eq. (6) represents evidently the pure TF approximation that is of lowest order in $\hbar$. In a first attempt and to assess the accuracy of our approximation, we will content ourselves with the TF approximation. Integration over the momenta yields the local density on the energy shell:

$$
\rho_{E}^{T F}(\boldsymbol{R})=\frac{1}{(2 \pi \hbar)^{3}} \int d \boldsymbol{p} f_{E}^{T F}(\boldsymbol{R}, \boldsymbol{p})=\frac{M k_{E}(\boldsymbol{R})}{2 \pi^{2} \hbar^{2} \widetilde{g}(E)},
$$

where

$$
k_{E}(\boldsymbol{R})=\sqrt{\frac{2 M}{\hbar^{2}}[E-V(\boldsymbol{R})]}
$$

is the local momentum at the energy $E$ in the potential $V(\boldsymbol{R})$, and the level density $\tilde{g}(E)$ is given by 

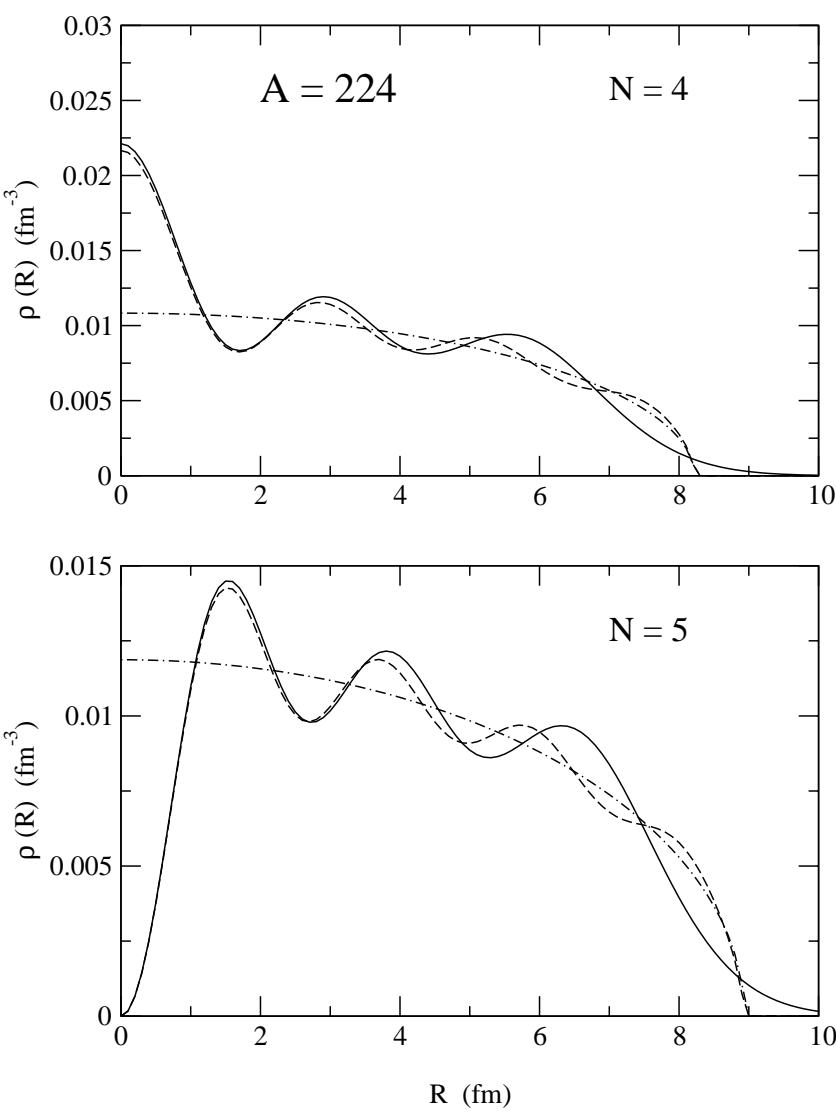

FIG. 1. Quantal (solid line), pure TF (dashed-dotted line), and TF densities projected on the good parity (dashed line) for the $N=4$ and $N=5$ harmonic oscillator shells.

$$
\tilde{g}(E)=\int d \boldsymbol{R} \frac{M k_{E}(\boldsymbol{R})}{2 \pi^{2} \hbar^{2}} .
$$

For the following, it is important to first elaborate on the meaning and accuracy of this density on the energy shell. For demonstration purposes, we will take as an example the spherical HO potential but later we will see that our method works equally well for a Woods-Saxon (WS) potential.

In Fig. 1 we display the quantal (solid line) and TF (dashdotted line) densities of the $N=4$ and $N=5 \mathrm{HO}$ shells with $\hbar \omega=41 A^{-1 / 3}$ and $A=224$. For the TF densities, we have taken the quantal energies. We see that in both cases the TF result passes accurately through the average, terminating at the classical turning point defined by

$$
E=V\left(\boldsymbol{R}_{c l}\right)
$$

The features of the TF densities on the energy shell are quite analogous to the ones already known for the full TF density [1]. However, on the quantal side one remarks that there is a strong difference between odd parity $(N=5)$ and even parity $(N=4)$ shells. The former show a pronounced hole at the origin whereas the second ones show, on the contrary, an enhancement. Both features can obviously be related to the absence or presence of the $s$-wave contributions in the corresponding $\mathrm{HO}$ shell, respectively. One may try to recover this even-odd parity effect in projecting the TF density matrix on good parity. This is easily done as follows. We calculate the inverse Wigner transform of $f_{E}^{T F}(\boldsymbol{R}, \boldsymbol{p})$. This yields

$$
\rho_{E}^{T F}\left(\boldsymbol{r}, \boldsymbol{r}^{\prime}\right)=\rho_{E}^{T F}(\boldsymbol{R}) j_{0}\left[s k_{E}(\boldsymbol{R})\right],
$$

where $j_{0}$ is the zeroth-order spherical Bessel function. Now the even-odd parity density on the energy shell is obtained as

$$
\begin{aligned}
\rho_{E}^{e / o}(\boldsymbol{r}) & =\frac{1}{2}\left[\rho_{E}^{T F}\left(\boldsymbol{r}, \boldsymbol{r}^{\prime}\right) \pm \rho_{E}^{T F}\left(\boldsymbol{r},-\boldsymbol{r}^{\prime}\right)\right]_{\boldsymbol{r}=\boldsymbol{r}^{\prime}} \\
& =\frac{1}{2}\left\{\rho_{E}^{T F}(\boldsymbol{r}) \pm \rho_{E}^{T F}(0) j_{0}\left[2 r k_{E}(0)\right]\right\} .
\end{aligned}
$$

We have drawn this expression in Fig. 1 (dashed lines) as well. The bump (hole) structure exhibited by the quantal density is now well reproduced in the interior. The agreement only deteriorates near the classical turning point. One should mention, however, that in spite of the seemingly rather spectacular improvement of formula (12) over Eq. (7), the former presents some small problems. This concerns the behavior of Eq. (12) around the turning point. The presence of the second term in Eq. (12) can induce a slightly negative value of the density around the turning point. Also the second term is not naturally limited to $r$ values inside the turning point and thus is oscillating around zero due to the Bessel function. This leads to ambiguities in evaluating matrix elements such as Eq. (2) which, however, numerically are rather unimportant. Thus we advocate to use Eq. (7) instead of Eq. (12), except for some problems where the even-odd bump structure may be particularly important. The latter may, for example, be the case for the evaluation of matrix elements of operators more concentrated at the nuclear interior, e.g. $1 / r^{2}$, etc.

\section{A. One-body matrix elements}

We now proceed to calculate in TF approximation, as a function of the energy, the rms radius of a nucleon confined in a WS potential with $V_{0}=-44 \mathrm{MeV}, a=0.67 \mathrm{fm}$, and $R$ $=1.27 A^{1 / 3} \mathrm{fm}$ with $A=224$ nucleons. We choose the rms radius for demonstration purposes but we could have taken as well any other smoothly varying single-particle operator. We use the TF approximation (11) and show the results (dashed line) together with their quantum mechanical counterparts, represented by dots, in Fig. 2. We see that the TF calculation very nicely passes through the average of the scattered quantal values, with the exception of the lowest $s$ state. This is a first confirmation of the accuracy of our approach.

In a next step we want to project the TF density matrix on different partial waves and calculate matrix elements as a function of the energy for different $l$ values. One way to project on partial waves has been elaborated by Hasse [17]. There one premultiplies the Wigner function with the semiclassical projectors on the orbital angular momentum and its $z$ component, i.e., 


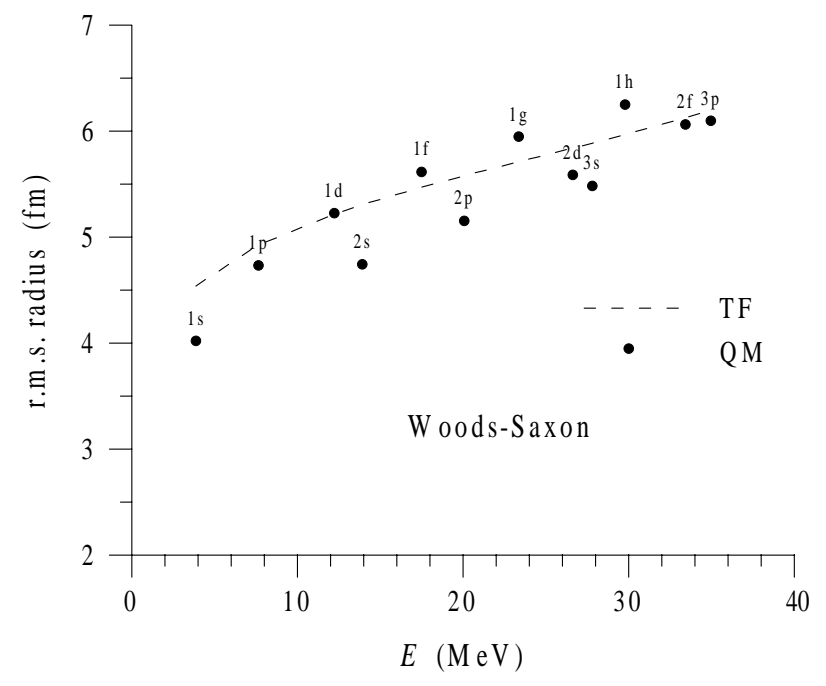

FIG. 2. Quantal and TF (dashed line) rms radii for a WoodsSaxon potential.

$f_{E, l, m}(\boldsymbol{R}, \boldsymbol{p})=\frac{1}{\tilde{g}_{E, l, m}} \delta(l-|\boldsymbol{R} \times \boldsymbol{p}|) \delta\left[m-l_{z}(R, p)\right] \delta\left(E-H_{c l}\right)$.

Then one can calculate single particle matrix elements as

$$
O(E, l, m)=\int \frac{d \boldsymbol{R} d \boldsymbol{p}}{(2 \pi \hbar)^{3}} O(\boldsymbol{R}, \boldsymbol{p}) f_{E, l, m}(\boldsymbol{R}, \boldsymbol{p}) .
$$

For local operators it is sufficient to know the density, which can be obtained in integrating Eq. (13) over momenta. Assuming spherical symmetry we can also sum over the $m$ quantum numbers, and after some algebra one finally finds [17]:

$$
\begin{aligned}
\rho_{E, l}(\boldsymbol{R})= & \frac{2 l+1}{8 \pi^{2} R^{2} \widetilde{g}_{E, l}} \sqrt{\frac{2 M}{\hbar^{2}}}\left[E-V(R)-\frac{\left(l+\frac{1}{2}\right)^{2} \hbar^{2}}{2 M R^{2}}\right]^{-1 / 2} \\
& \times \Theta\left(E-V(R)-\frac{\left(l+\frac{1}{2}\right)^{2} \hbar^{2}}{2 M R^{2}}\right)
\end{aligned}
$$

where the level density $\tilde{g}_{E, l}$ is chosen in such a way that the integral of Eq. (15) over the available $R$ space is normalized to unity. Let us mention that we have replaced $l(l+1)$ by $\left(l+\frac{1}{2}\right)^{2}$, as it is done in the WKB method to recover the right asymptotic behavior of the wave function in the free $[V(R)=0]$ case [18].

We recently employed, however, a different way to do the $l$ projection, which for some purposes may be more convenient [19]. For this we first perform the inverse Wigner transform of the TF part of Eq. (6),

$$
\begin{aligned}
\rho_{E}\left(\boldsymbol{r}, \boldsymbol{r}^{\prime}\right)= & \frac{1}{\widetilde{g}(E)} \int \frac{d \boldsymbol{p}}{(2 \pi \hbar)^{3}} e^{i \boldsymbol{p} \cdot \boldsymbol{r} / \hbar} e^{-i \boldsymbol{p} \cdot \boldsymbol{r}^{\prime} / \hbar} \\
& \times \delta\left(E-\frac{p^{2}}{2 M}-V(\boldsymbol{R})\right) .
\end{aligned}
$$

Expanding the plane waves in spherical harmonics,

$$
e^{i k \cdot r}=4 \pi \sum_{l, m}(-i)^{l} j_{l}(k r) Y_{l m}\left(\Omega_{k}\right) Y_{l m}^{*}\left(\Omega_{r}\right),
$$

we can read off the $l$-projected density matrix. For the local density, we then obtain

$$
\begin{aligned}
\rho_{E, l, m}(\boldsymbol{R})= & \frac{1}{\tilde{g}_{E, l, m}} \frac{2 M}{\pi \hbar^{2}} k_{E}(R)\left\{j_{l}\left[R k_{E}(R)\right]\right\}^{2}\left|Y_{l m}(\Omega)\right|^{2} \\
& \times \Theta[E-V(\boldsymbol{R})],
\end{aligned}
$$

where $\tilde{g}_{E, l, m}$ is again chosen to ensure the right normalization of Eq. (18), i.e., $\int d \boldsymbol{R} \rho_{E, l, m}(\boldsymbol{R})=1$. We can calculate, for example, the mean square radius as a function of $E$ for different $l$ values,

$$
\left\langle R^{2}\right\rangle_{E, l}=\int d \boldsymbol{R} R^{2} \rho_{E, l, m}(\boldsymbol{R}) .
$$

Notice that Eq. (19) becomes independent of $m$ after the angular integration.

In Table I we show various moments $\left\langle R^{n}\right\rangle^{1 / n}$ (in fm) obtained using the TF local densities on the energy shell provided by Eqs. (15) and (18), as compared with the corresponding quantal values for the aforementioned WS potential with $A=224$ nucleons. From these tables one can see that the quantal rms radius $(n=2)$ of each $n l$ state is, on average, well reproduced using the semiclassical $l$-projected on-shell densities given by the TF approaches in Eqs. (15) and (18), where $E$ has been replaced by the quantal eigenvalue corresponding to the $n l$ state (for consistency, we should perform a WKB quantization from which we refrain for simplicity as it would not affect the result much). A more quantitative analysis shows that the quantal rms radii are reproduced by Eq. (15) within $2 \%$ in average over the range of energies considered, while using Eq. (18) the average relative error is around $4 \%$. Higher moments obtained with the TF densities on the energy shell also reproduce reasonably well the results of the full quantal calculation. For the highest moment considered here $(n=10)$, the average relative error with respect to the quantal calculation is around $4.3 \%$ for both semiclassical approximations. One should point out that the TF local densities on the energy shell are free of the shell effects that are present in the quantal calculation. Actually, the TF results represent the shell averaged values of the moments and their difference with the quantal calculations provides an estimate of the shell correction for the considered state. Of course, a precise calculation of the shell correction in the considered moments would require a Strutinsky calculation, which is not an easy task for a WS potential. For a related discussion we refer the reader to Ref. [20], where the moments ranging 
TABLE I. Moments $\left\langle R^{n}\right\rangle^{1 / n}$ (in fm) of several $n l$ states for $A=224$ particles in the Woods-Saxon potential described in the text. The full quantum mechanical values (QM) are compared with those obtained with the semiclassical approaches, Eqs. (15) and (18).

\begin{tabular}{|c|c|c|c|c|c|c|c|}
\hline$n l$ & & $E(\mathrm{MeV})$ & $n=2$ & $n=4$ & $n=6$ & $n=8$ & $n=10$ \\
\hline \multirow[t]{3}{*}{$1 s$} & $\mathrm{QM}$ & 3.87 & 4.021 & 4.441 & 4.780 & 5.069 & 5.323 \\
\hline & Eq. (15) & & 4.040 & 4.528 & 4.824 & 5.019 & 5.160 \\
\hline & Eq. (18) & & 4.299 & 4.638 & 4.863 & 5.024 & 5.143 \\
\hline \multirow[t]{3}{*}{$1 p$} & QM & 7.65 & 4.732 & 5.042 & 5.307 & 5.543 & 5.758 \\
\hline & Eq. (15) & & 4.653 & 5.011 & 5.267 & 5.449 & 5.583 \\
\hline & Eq. (18) & & 4.900 & 5.144 & 5.321 & 5.546 & 5.561 \\
\hline \multirow[t]{3}{*}{$1 d$} & $\mathrm{QM}$ & 12.23 & 5.226 & 5.477 & 5.701 & 5.907 & 6.099 \\
\hline & Eq. (15) & & 5.123 & 5.397 & 5.612 & 5.776 & 5.900 \\
\hline & Eq. (18) & & 5.323 & 5.516 & 5.664 & 5.782 & 5.876 \\
\hline \multirow[t]{3}{*}{$2 s$} & QM & 13.92 & 4.746 & 5.401 & 5.780 & 6.052 & 6.275 \\
\hline & Eq. (15) & & 4.668 & 5.301 & 5.662 & 5.897 & 6.064 \\
\hline & Eq. (18) & & 4.683 & 5.331 & 5.679 & 5.901 & 6.060 \\
\hline \multirow[t]{3}{*}{$1 f$} & QM & 17.49 & 5.616 & 5.831 & 6.031 & 6.218 & 6.397 \\
\hline & Eq. (15) & & 5.511 & 5.731 & 5.913 & 6.057 & 6.172 \\
\hline & Eq. (18) & & 5.568 & 5.819 & 5.948 & 6.054 & 6.141 \\
\hline \multirow[t]{3}{*}{$2 p$} & QM & 20.08 & 5.154 & 5.771 & 6.159 & 6.439 & 6.668 \\
\hline & Eq. (15) & & 5.065 & 5.653 & 6.009 & 6.244 & 6.411 \\
\hline & Eq. (18) & & 4.931 & 5.601 & 6.002 & 6.256 & 6.433 \\
\hline \multirow[t]{3}{*}{$1 g$} & QM & 23.37 & 5.948 & 6.143 & 6.327 & 6.504 & 6.679 \\
\hline & Eq. (15) & & 5.836 & 6.021 & 6.179 & 6.309 & 6.414 \\
\hline & Eq. (18) & & 5.942 & 6.083 & 6.200 & 6.297 & 6.379 \\
\hline \multirow[t]{3}{*}{$2 d$} & $\mathrm{QM}$ & 26.63 & 5.587 & 6.159 & 6.547 & 6.836 & 7.078 \\
\hline & Eq. (15) & & 5.480 & 6.014 & 6.354 & 6.583 & 6.747 \\
\hline & Eq. (18) & & 5.293 & 5.942 & 6.356 & 6.622 & 6.802 \\
\hline \multirow[t]{3}{*}{$3 s$} & $\mathrm{QM}$ & 27.80 & 5.485 & 6.236 & 6.679 & 6.998 & 7.263 \\
\hline & Eq. (15) & & 5.374 & 6.077 & 6.467 & 6.719 & 6.895 \\
\hline & Eq. (18) & & 5.156 & 6.014 & 6.483 & 6.771 & 6.963 \\
\hline \multirow[t]{3}{*}{$1 h$} & QM & 29.78 & 6.252 & 6.435 & 6.612 & 6.788 & 6.967 \\
\hline & Eq. (15) & & 6.128 & 6.289 & 6.429 & 6.547 & 6.644 \\
\hline & Eq. (18) & & 6.177 & 6.316 & 6.427 & 6.521 & 6.601 \\
\hline \multirow[t]{3}{*}{$2 f$} & QM & 33.44 & 6.063 & 6.606 & 6.999 & 7.304 & 7.584 \\
\hline & Eq. (15) & & 5.923 & 6.410 & 6.731 & 6.950 & 7.108 \\
\hline & Eq. (18) & & 5.775 & 6.375 & 6.766 & 7.018 & 7.188 \\
\hline \multirow[t]{3}{*}{$3 p$} & QM & 34.97 & 6.097 & 6.862 & 7.341 & 7.709 & 8.037 \\
\hline & Eq. (15) & & 5.939 & 6.621 & 7.004 & 7.421 & 7.421 \\
\hline & Eq. (18) & & 5.866 & 6.621 & 7.036 & 7.294 & 7.469 \\
\hline
\end{tabular}

from $n=1$ to $n=10$ of the total density of $A=224$ particles in a $\mathrm{HO}$ potential were evaluated using several semiclassical approaches and the Strutinsky averaging method. The fact that TF works for moments as high as $n=10$ makes us believe that one can certainly consider in our approach all single-particle operators that are low-order polynomials in the phase-space variables.

It is also instructive to directly compare the densities. For this, we again take $E$ equal to its quantal value in Eqs. (15) and (18). The comparison between the quantal and TF onshell densities is shown in Figs. 3-5. In each one of these figures, the quantal on-shell density (solid line) and the semiclassical densities provided by Eq. (15) (dash-dotted line) and Eq. (18) (dashed line) are displayed for all the bound $s$ and $p$ states of a WS potential with $A=224$. From these figures one can see that the quantal on-shell densities are rather well reproduced by the TF approach, Eq. (18). In particular, the quantal $1 l$ on-shell densities are well reproduced by Eq. (18) in the interior. However, as in Fig. 1, there are discrepancies between the quantal and TF densities in the outer part around the classical turning point. For $n l$ states $(n>1)$, the inner $n-1$ bumps are well reproduced by Eq. (18), and the agreement deteriorates in the outer bumps due to the classical turning point where the TF densities vanish. On the other hand, the TF on-shell densities obtained with Eq. (15) do not reproduce the quantal density profiles at all. As it can be seen from Eq. (15), this density is defined in the region in between the two roots (turning points) of 

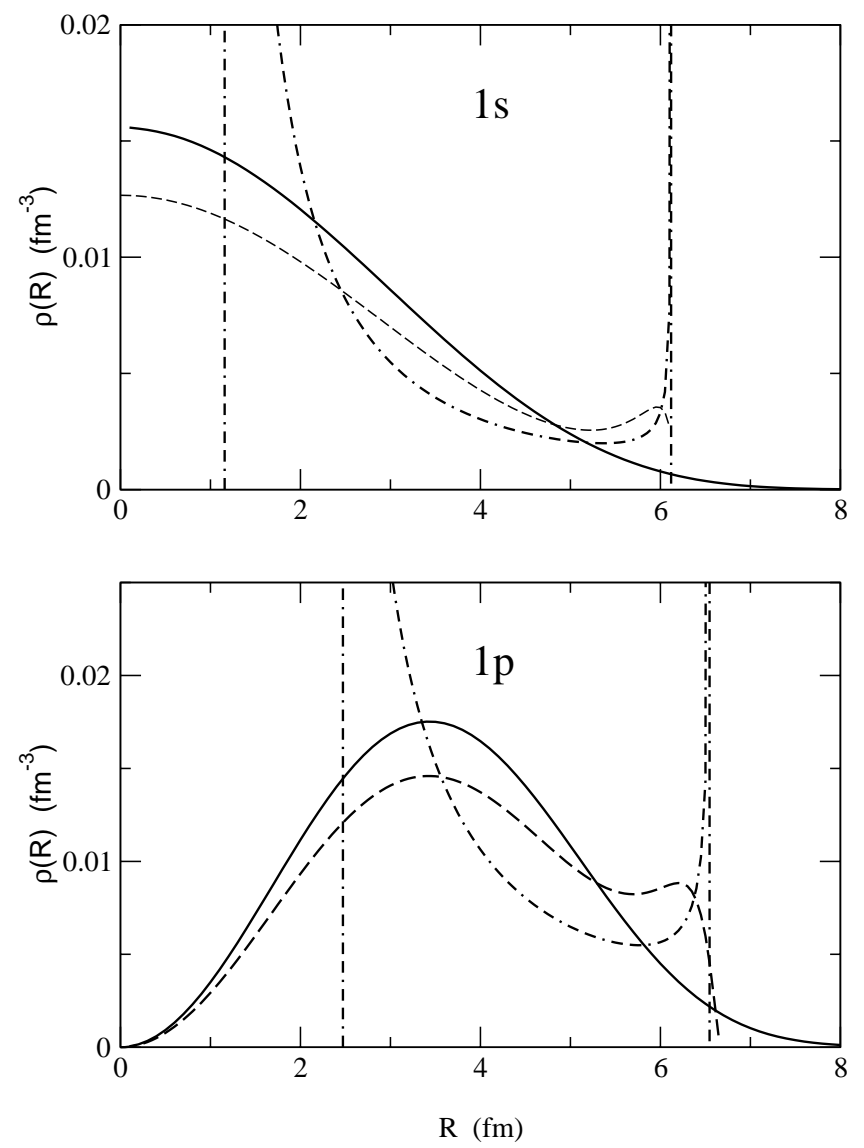

FIG. 3. $1 s$ and $1 p$ on-shell densities in a Woods-Saxon potential calculated quantally (solid line) and with the TF approximation using Eq. (15) (dash-dotted line) and Eq. (18) (dashed line). The dashdotted vertical lines indicate the asymptotes of Eq. (15).

$$
E-V(R)-\frac{\left(l+\frac{1}{2}\right)^{2} \hbar^{2}}{2 M R^{2}}=0
$$

In this approach, the three-dimensional problem has been reduced to an equivalent one-dimensional problem for $R$, with an effective potential that in addition to $V(R)$ contains the centrifugal barrier, as it happens in the WKB method. Thus, in this case, we find two different turning points. The largest root of Eq. (20) is very close to the classical turning point of Eq. (18) given by $k_{E}=0$ [see Eq. (8)], while the smallest root gives the inner turning point due to the centrifugal barrier. Since the TF on-shell density (15) has square root singularities at the two turning points, its integral as well as the corresponding expectation values converge.

We arrive at the, at first sight, paradoxical result that the densities (15) that have no detailed resemblance with the quantal ones reproduce the rms values (and very likely most of other expectation values of smoothly varying operators) better than the densities given in Eq. (18), which show quite reasonable overall behavior in comparison with the quantal results. We here find an illustrating example that the ThomasFermi and Wigner-Kirkwood local densities are to be regarded as mathematical distributions, in the sense that in
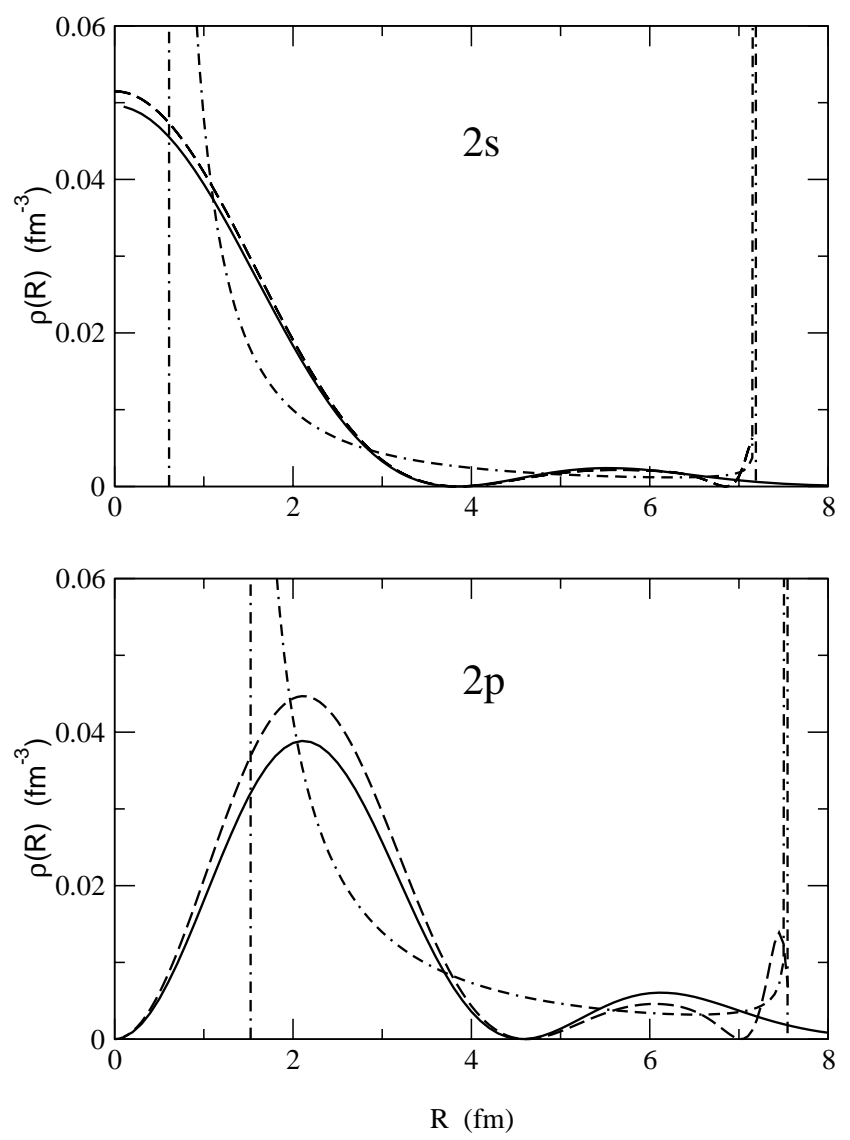

FIG. 4. Same as Fig. 3 for the $2 s$ and $2 p$ on-shell densities.

spite of their possible divergences, they yield finite and accurate results when used to compute a restricted class of expectation values by integration $[13,20]$. In this respect, we again refer the reader to Tables I and II where he finds confirmation of our statement. A similar situation is found in computing the kinetic energy for a bosonic system in Ref. [16]: the semiclassical and quantal kinetic energy densities are clearly different but give similar values of the integrated kinetic energy. On the other hand, one should note that sorting out correctly the various orders in $\hbar$ is very important to achieve optimal results as shown on other occasions [20,21]. In expression (18), there remain some $\hbar$ corrections to all orders in the form of the spherical harmonics which are quantal wave functions, i.e., solutions of a Schrödinger equation. This mixing of resummation in $\hbar$ on the one hand and of lowest order in $\hbar$ on the other hand [in the form of the $\delta$ function in Eq. (16)] finally makes the on-shell density (18) slightly less accurate than Eq. (15), which represents the correct $\hbar \rightarrow 0$ limit as shown in Ref. [17].

\section{B. Two-body matrix elements}

As a further interesting application we want to consider the semiclassical evaluation of average two-body matrix elements. An example of particular interest is the case of matrix elements of the pairing type, $\left\langle\Phi(\nu, \bar{\nu})|v| \Phi\left(\nu^{\prime}, \bar{\nu}^{\prime}\right)\right\rangle$ [see 

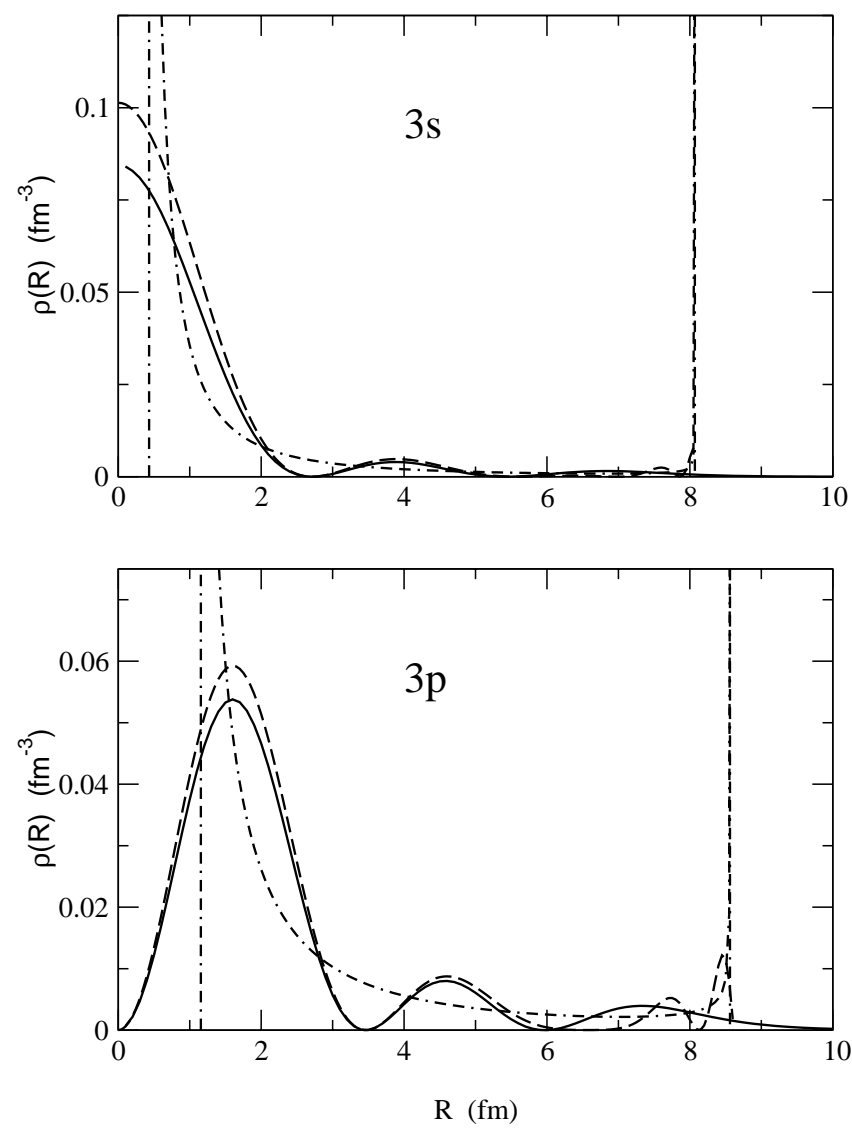

FIG. 5. Same as Fig. 3 for the $3 s$ and $3 p$ on-shell densities.

Eq. (4)], which we shall address in this section for identical nucleons. It is straightforward to recast the state-dependent pairing matrix element as

$$
\left\langle\Phi(\nu, \bar{\nu})|v| \Phi\left(\nu^{\prime}, \bar{\nu}^{\prime}\right)\right\rangle=\left\langle\nu \bar{\nu}|v| \nu^{\prime} \bar{\nu}^{\prime}\right\rangle-\left\langle\nu \bar{\nu}|v| \bar{\nu}^{\prime} \nu^{\prime}\right\rangle .
$$

The two-particle states $|\nu \bar{\nu}\rangle$ on the right-hand side are product states of $|\nu\rangle$ and $|\bar{\nu}\rangle$. The states $|\nu\rangle$ are represented by single-particle wave functions $\phi_{\nu}(\boldsymbol{r}, \sigma)=\phi_{n l m}(\boldsymbol{r}) \chi_{\sigma}$ (with $\left.\sigma= \pm \frac{1}{2}\right)$. Assuming spherical symmetry, and considering that the time reversal of $|\nu\rangle$ involves $\hat{T}\left(Y_{l m} \chi_{\sigma}\right)$ $=(-1)^{m} Y_{l,-m}(-1)^{1 / 2-\sigma} \chi_{-\sigma}=(-1)^{1 / 2-\sigma} Y_{l m}^{*} \chi_{-\sigma}$, one finds

$$
\begin{aligned}
\frac{1}{4} \sum_{m, m^{\prime}} & \sum_{\sigma, \sigma^{\prime}}\left\langle\Phi(\nu, \bar{\nu})|v| \Phi\left(\nu^{\prime}, \bar{\nu}^{\prime}\right)\right\rangle \\
= & \sum_{m, m^{\prime}} \int d \boldsymbol{r} d \boldsymbol{r}^{\prime} \phi_{n l m}\left(\boldsymbol{r}^{\prime}\right) \phi_{n l m}^{*}(\boldsymbol{r}) v\left(\boldsymbol{r}-\boldsymbol{r}^{\prime}\right) \\
& \times \phi_{n^{\prime} l^{\prime} m^{\prime}}(\boldsymbol{r}) \phi_{n^{\prime} l^{\prime} m^{\prime}}^{*}\left(\boldsymbol{r}^{\prime}\right) .
\end{aligned}
$$

According to this result, we obtain the following expression for the average pairing matrix elements of Eq. (4):

$$
v\left(E, E^{\prime}\right)=\int d \boldsymbol{r} d \boldsymbol{r}^{\prime} \rho_{E}\left(\boldsymbol{r}, \boldsymbol{r}^{\prime}\right) v\left(\boldsymbol{r}-\boldsymbol{r}^{\prime}\right) \rho_{E^{\prime}}\left(\boldsymbol{r}^{\prime}, \boldsymbol{r}\right),
$$

where $\rho_{E}\left(\boldsymbol{r}, \boldsymbol{r}^{\prime}\right)=\left\langle\boldsymbol{r}\left|\hat{\rho}_{E}\right| \boldsymbol{r}^{\prime}\right\rangle$. In TF approximation, the nonlocal on-shell density matrix $\rho_{E}\left(\boldsymbol{r}, \boldsymbol{r}^{\prime}\right)$ is given by Eq. (11). We see that it is a symmetric function in $\boldsymbol{r}$ and $\boldsymbol{r}^{\prime}$.

For the case of a force $v_{0} \delta\left(\boldsymbol{r}-\boldsymbol{r}^{\prime}\right)$, Eq. (23) reduces to (a practically identical expression can be found in Ref. [22])

$$
v\left(E, E^{\prime}\right)=v_{0} \int d \boldsymbol{r} \rho_{E}(\boldsymbol{r}) \rho_{E^{\prime}}(\boldsymbol{r})
$$

Using the TF expression (7) for $\rho_{E}(\boldsymbol{r})$, we can evaluate Eq. (24) with a HO potential $V(\boldsymbol{r})=m \omega_{0}^{2} r^{2} / 2$ and compare with the quantum mechanical matrix elements averaged on each major shell $N$ of energy $E=E_{N}=\left(N+\frac{3}{2}\right) \hbar \omega$. This is done in Table II with $v_{0}=-345.723 \mathrm{MeV} \mathrm{fm}^{3}$ and $\hbar \omega_{0}=41 A^{-1 / 3}$ $\mathrm{MeV}$. We again see that the semiclassical results agree very well with the averaged quantal values, even for the nondiagonal elements.

With the above positive experience at hand, we next proceed to calculate the average pairing matrix elements $v\left(\varepsilon_{F}, \varepsilon_{F}\right)$ of the Gogny D1S force [23] that is known to

TABLE II. QM and TF averaged two-body matrix elements (in MeV) of the $v\left(\boldsymbol{r}, \boldsymbol{r}^{\prime}\right)=-345.723 \delta(\boldsymbol{r}$ $-\boldsymbol{r}^{\prime}$ ) force calculated with harmonic oscillator density matrices on the energy shell for $A=224$ particles.

\begin{tabular}{lccccccc}
\hline \hline & $N / N^{\prime}$ & 0 & 1 & 2 & 3 & 4 & 5 \\
\hline QM & 0 & -1.44 & -0.72 & -0.45 & -0.32 & -0.24 & -0.19 \\
TF & & -1.20 & -0.68 & -0.44 & -0.31 & -0.23 & -0.18 \\
QM & 1 & & -0.60 & -0.41 & -0.29 & -0.22 & -0.18 \\
TF & & & -0.56 & -0.39 & -0.29 & -0.22 & -0.18 \\
QM & 2 & & & -0.35 & -0.27 & -0.21 & -0.17 \\
TF & & & & -0.34 & -0.26 & -0.21 & -0.17 \\
QM & 3 & & & & -0.24 & -0.19 & -0.16 \\
TF & & & & & -0.23 & -0.19 & -0.16 \\
QM & 4 & & & & & -0.17 & -0.15 \\
TF & & & & & & -0.17 & -0.15 \\
QM & 5 & & & & & & -0.14 \\
TF & & & & & & & \\
\hline \hline
\end{tabular}


reproduce experimental gap values when used in microscopic Hartree-Fock-Bogolyubov calculations [24]. Writing the diagonal matrix element (4) at the Fermi energy $\varepsilon_{F}$, by using Eq. (23), and expressing it through the lowest-order Wigner function in inverting Eq. (5), one arrives in TF approximation at

$$
\begin{aligned}
v\left(\varepsilon_{F}, \varepsilon_{F}\right)= & \frac{1}{\tilde{g}\left(\varepsilon_{F}\right)^{2}} \int d \boldsymbol{R} \int \frac{d \boldsymbol{p} d \boldsymbol{p}^{\prime}}{(2 \pi \hbar)^{6}} \delta\left[\varepsilon_{F}-H_{c l}(\boldsymbol{R}, \boldsymbol{p})\right] \\
& \times v\left(\boldsymbol{p}-\boldsymbol{p}^{\prime}\right) \delta\left[\varepsilon_{F}-H_{c l}\left(\boldsymbol{R}, \boldsymbol{p}^{\prime}\right)\right] .
\end{aligned}
$$

Here, $H_{c l}=p^{2} / 2 M^{*}+V(\boldsymbol{R})$ is the classical Hamiltonian of independent particles with effective mass $M^{*}$ (see below) moving in an external potential well $V(\boldsymbol{R})$, and $v\left(\boldsymbol{p}-\boldsymbol{p}^{\prime}\right)$ is the Fourier transform of the particle-particle part of the Gogny force which describes the pairing. For the numerical application, we use for $V(\boldsymbol{R})$ a slight variant of the potential given by Shlomo [14]:

$$
V(R)=-\frac{V_{0}}{1+\exp \left(-R_{0} / d\right)}+\frac{V_{0}}{1+\exp \left[\left(R-R_{0}\right) / d\right]},
$$

with

$$
\begin{gathered}
R_{0}=\frac{1.12 A^{1 / 3}+1}{\left[1+\left(\pi d / R_{0}\right)^{2}\right]^{1 / 3}} \mathrm{fm}, \\
d=0.70 \mathrm{fm}, \quad \text { and } \quad V_{0}=-54 \mathrm{MeV} .
\end{gathered}
$$

In this equation, $R_{0}$ has to be determined iteratively.

Equation (25) can be reduced to a one dimensional integral over $R$, which can be performed numerically,

$$
\begin{aligned}
v\left(\varepsilon_{F}, \varepsilon_{F}\right)= & \sum_{c=1}^{2} z_{c} \frac{1}{\mu_{c}^{2}} \int_{0}^{R_{c}} d R R^{2} B \\
& \times \exp \left\{-a\left[\varepsilon_{F}-V(R)\right]\right\} \sinh \left\{a\left[\varepsilon_{F}-V(R)\right]\right\},
\end{aligned}
$$

where $R_{c}$ is the classical turning point defined in Eq. (10), and

$$
B=\frac{1}{4 \pi^{3} \widetilde{g}\left(\varepsilon_{F}\right)^{2}}\left(\frac{2 M^{*}}{\hbar^{2}}\right)^{2}, \quad a=\frac{M^{*} \mu_{c}^{2}}{\hbar^{2}},
$$

and

$$
z_{c}=\pi^{3 / 2} \mu_{c}^{3}\left(W_{c}-B_{c}-H_{c}+M_{c}\right) .
$$

The factors $z_{c}$ correspond to pairing in the $S=0$ and $T=1$ channels and are written in terms of the parameters of the Gogny force $\left(W_{c}, B_{c}, H_{c}, M_{c}\right.$, and $\left.\mu_{c}\right)$ [23]. We have introduced the position-dependent effective mass $M^{*}(\boldsymbol{R})$ from the Gogny force in order to make the calculation of the pairing matrix element more realistic. It is obtained by evaluating at $k=k_{\varepsilon_{F}}(\boldsymbol{R})$ the position- and momentum-dependent effective mass [25],

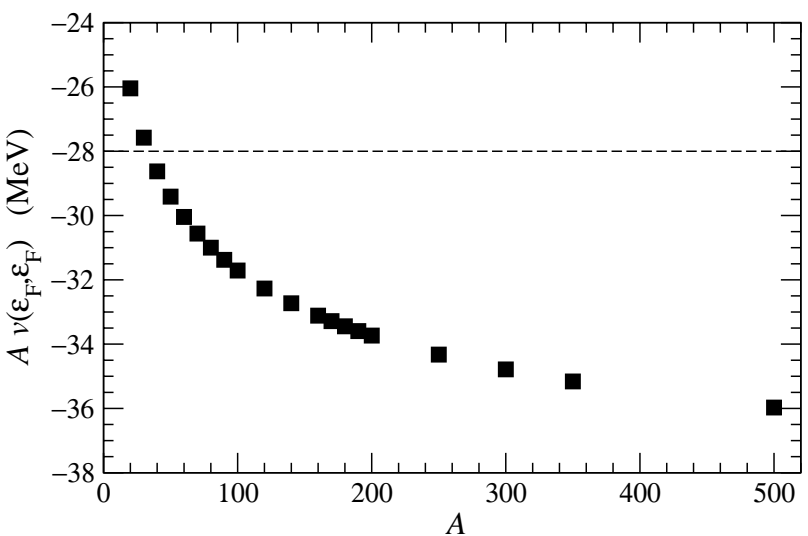

FIG. 6. Two-body pairing matrix elements computed with the D1S Gogny force and the Shlomo potential (26) as a function of the mass number $A$.

$$
\frac{M}{M^{*}(\boldsymbol{R}, k)}=1+\frac{M}{\hbar^{2} k} \frac{\partial}{\partial k} U(\boldsymbol{R}, k)
$$

where $U(\boldsymbol{R}, k)$ is the Wigner transform of the single-particle potential obtained from the Gogny interaction assumed spherically symmetric in $\boldsymbol{k}$.

Also, the level density $\tilde{g}\left(\varepsilon_{F}\right)$ is calculated in the TF approach using the same potential and effective mass,

$$
\tilde{g}\left(\varepsilon_{F}\right)=\frac{1}{\pi} \int_{0}^{R_{C}} d R R^{2}\left(\frac{2 M^{*}(\boldsymbol{R})}{\hbar^{2}}\right)^{3 / 2} \sqrt{\varepsilon_{F}-V(\boldsymbol{R})} .
$$

In Fig. 6, we show $A v\left(\varepsilon_{F}, \varepsilon_{F}\right)$ as a function of the mass number $A$. The Coulomb force has been switched off in the present calculation. We see that there is a quite pronounced $A$ dependence, which is somewhat in contradiction with the value $G \sim 28 / \mathrm{A} \mathrm{MeV}$ for the constant pairing matrix element at the Fermi level usually employed in more schematic treatments of the nuclear pairing. On the other hand, if we calculate $A v\left(\varepsilon_{F}, \varepsilon_{F}\right)$ not with a WS potential but with the $\mathrm{HO}$ potential, we obtain a practically constant value. This may indicate that the $A^{-1}$ dependence of the constant pairing matrix element is better fulfilled in conjunction with a harmonic potential. The difference in the behavior with $A$ using the $\mathrm{HO}$ and WS potentials may come from the absence (HO) or presence (WS) of a surface contribution to $v\left(\varepsilon_{F}, \varepsilon_{F}\right)$, like it is the case for the level density [1].

\section{CONCLUSIONS}

In this work we showed how average nuclear one- and two-body matrix elements can very efficiently be evaluated using the Thomas-Fermi approach. The main ingredient is to replace the density matrix for a given quantum state $\hat{\rho}_{\nu}$ $=|\nu\rangle\langle\nu|$ by its counterpart averaged over the energy shell,

$$
\hat{\rho}_{E}=\frac{1}{\widetilde{g}(E)} \sum_{\nu} \widetilde{\delta}\left(E-\varepsilon_{\nu}\right)|\nu\rangle\langle\nu|,
$$


with simultaneous application of the Wigner-Kirkwood $\hbar$ expansion for the smoothly varying spectral density $\widetilde{\delta}(E-\hat{H})$ and level density $\widetilde{g}(E)=\operatorname{Tr}[\widetilde{\delta}(E-\hat{H})]$.

We calculated one- and two-body matrix elements, restricting ourselves (in this exploratory work) to the lowest order, i.e., the pure Thomas-Fermi approximation. We compared quantal and semiclassical values of the matrix elements using harmonic oscillator and Woods-Saxon type of potentials. We did this also for parity projected and for angular momentum projected Thomas-Fermi theory. In all the cases close agreement with the average quantal behaviors was found, showing the accuracy of the method. As in the case of the well-tested Wigner-Kirkwood expansion of the full density matrix [1], one expects that some improvement also could be achieved for the matrix elements by inclusion of $\hbar$ corrections.

With the positive result for the single-particle matrix elements at hand, we also calculated the average pairing matrix elements of some effective nuclear two-body forces. First, we used a $\delta$ interaction and compared diagonal and offdiagonal semiclassical elements with the corresponding quantal values. Again, the TF values nicely reproduce the quantum results on average. Next we estimated semiclassically the diagonal pairing matrix elements of the Gogny D1S force at the Fermi energy. Since the Gogny force is known to reproduce very well nuclear pairing properties [24], it is interesting to evaluate, e.g., the $A$ dependence of its pairing matrix elements around the Fermi energy and to see to which extent the common assumption of a $A^{-1}$ dependence holds. Using for the mean field a potential of the Woods-Saxon type, it turned out that the falloff of the pairing matrix ele- ment is stronger than the $A^{-1}$ law. For this problem we have no comparison with quantum values available, but the experience with the one-body matrix elements and the pairing matrix elements for the $\delta$ force makes us believe that the values shown in Fig. 6 are also reasonably accurate. The stronger than $A^{-1}$ decrease observed in Fig. 6 has its origin very likely in the presence of a surface contribution implicit from the use of a WS potential, whereas the use of a $\mathrm{HO}$ potential with the absence of a surface shows agreement with the $A^{-1}$ law.

It should be emphasized that, to our knowledge, the TF method to calculate average matrix elements of a two-body force has never been applied before. We think that the conclusive study of this work will allow one to use average matrix elements for the calculation of many nuclear quantities where fine shell effects are not needed, such as optical potentials, giant resonances and their widths, and many other quantities where the average trend is of interest. In a future publication, we will show how the application of these techniques can be used to study in a very transparent way the size dependence of the average pairing gap in finite Fermi systems in an almost analytical way.

\section{ACKNOWLEDGMENTS}

P. S. wants to thank P. Leboeuf and N. Pavloff for useful discussions and information. Two of us (X. V. and M. C.) acknowledge financial support from the DGI (Ministerio de Ciencia y Tecnología, Spain) and the FEDER under Grant No. BFM2002-01868 and from the DGR (Catalonia) under Grant No. 2001SGR-00064.
[1] P. Ring and P. Schuck, The Nuclear Many-Body Problem (Springer-Verlag, New York, 1980); M. Brack and R.K. Badhuri, Semiclassical Physics (Addison-Wesley, Reading, MA, 1997).

[2] M. Brack and H.C. Pauli, Nucl. Phys. A207, 401 (1973); B.K. Jennings, ibid. A207, 538 (1973).

[3] R.W. Hasse and P. Schuck, Phys. Lett. B 179, 313 (1986).

[4] M. Durand, P. Schuck, and X. Viñas, Z. Phys. A 346, 87 (1993); M. Centelles, X. Viñas, and P. Schuck, Phys. Rev. C 53, 1018 (1996).

[5] R.K. Bhaduri and C.K. Ross, Phys. Rev. Lett. 27, 606 (1971); B.K. Jennings and R.K. Bhaduri, Nucl. Phys. A237, 149 (1975); B.K. Jennings, R.K. Bhaduri, and M. Brack, Phys. Rev. Lett. 34, 228 (1975); Nucl. Phys. A253, 29 (1975).

[6] K. Taruishi and P. Schuck, Z. Phys. A 342, 397 (1992); P. Schuck and K. Taruishi, Phys. Lett. B 385, 12 (1996).

[7] M. Brack, J. Damgård, A.S. Jensen, H.C. Pauli, V.M. Strutinsky, and C.Y. Wong, Rev. Mod. Phys. 44, 320 (1972).

[8] M. Wilkinson, J. Phys. A 20, 2415 (1987).

[9] B. Mehlig, Phys. Rev. E 59, 390 (1999).

[10] V.N. Kondratyev, Phys. Lett. A 179, 209 (1993).

[11] M.L. Du and J.B. Delos, Phys. Rev. A 38, 1896 (1988); 38,
1913 (1988).

[12] X. Viñas, P. Schuck, M. Farine, M. Durand, and M. Centelles, Phys. At. Nucl. 65, 731 (2002); Yad. Fiz. 65, 764 (2002).

[13] H. Krivine, M. Casas, and J. Martorell, Ann. Phys. (N.Y.) 200, 304 (1990).

[14] S. Shlomo, Nucl. Phys. A539, 17 (1992).

[15] A.B. Migdal, Nucl. Phys. 13, 655 (1959).

[16] P. Schuck and X. Viñas, Phys. Rev. A 61, 043603 (2000).

[17] R.W. Hasse, Ann. Univ. Mariae Curie Sklodowska [Med] XL/ XLI, 191 (1965); Nucl. Phys. A467, 407 (1987).

[18] L. D. Landau and E. M. Lifshitz, Quantum Mechanics (Pergamon, New York, 1991).

[19] M. Urban, P. Schuck, and X. Vinas, cond-mat/0207261.

[20] M. Centelles, X. Viñas, M. Durand, P. Schuck, and D. VonEiff, Ann. Phys. (N.Y.) 266, 207 (1998).

[21] P. Schuck and X. Viñas, Phys. Lett. B 302, 1 (1993).

[22] F. Tondeur, Nucl. Phys. A315, 353 (1979).

[23] J.F. Berger, M. Girod, and D. Gogny, Comput. Phys. Commun. 63, 365 (1991); Nucl. Phys. A502, 85c (1989).

[24] M. Kleban, B. Nerlo-Pomorska, J.F. Berger, J. Dechargé, M. Girod, and S. Hilaire, Phys. Rev. C 65, 024309 (2002).

[25] V.B. Soubbotin and X. Viñas, Nucl. Phys. A665, 291 (2000). 